\title{
El influjo norteamericano en el desarrollo de la salud pública en Colombia
}

\author{
Juan Carlos Eslava
}

\begin{abstract}
Resumen
El presente artículo intenta mostrar cómo la salud pública colombiana, al igual que la del resto de Latinoamérica, se desarrolló bajo la profunda influencia de los modelos y las pautas norteamericanas, pero condicionada, a su vez, por las particularidades nacionales. Esto permite reflexionar sobre los procesos diferenciales mediante los cuales las políticas del "centro", representado por las directrices de la Fundación Rockefeller, influyen los espacios "periféricos". La hipótesis de trabajo que se propone para explicar la particularidad del caso colombiano, en el contexto latinoamericano, es la ausencia de un movimiento nacionalista significativo de comienzos de siglo que contribuya a forjar un movimiento sanitarista fuerte y enraizado en lo local y con posibilidades de entablar negociaciones claras y contundentes frente a las posturas norteamericanas, lo que hace que la salud pública colombiana adopte una forma diferente y más dependiente que la de otros países de la región.
\end{abstract}

\section{North American influence on the development of public health in Colombia}

The present article tries to show how Colombian public health, the same as in the rest of Latin America, was developed under the profound influence of North American models and norms, but conditioned, in turn, by national particularities. This allows reflection about the differential processes through which the policies of the "centre", represented by the Rockefeller Foundation's directives, influenced the "peripheral" spaces. The working hypothesis which is proposed to explain the Colombian case's particularity, in the Latin American context, is the absence of a significant nationalist movement at the start of the century which could have contributed towards forging a strong sanitary movement rooted in that which was local and having the possibility to table clear and convincing negotiation faced with North American posturing, as what did happen was that Colombian public health took on a different and more dependent form than that of the region's other countries.

El presente trabajo se inscribe dentro del proyecto "El tránsito de la higiene a la medicina tropical y la salud pública en Colombia", el cual es dirigido por Emilio Quevedo y cuenta con el apoyo del Instituto Nacional de Salud, la Universidad Nacional y COLCIENCIAS. La versión original del texto fue presentada en el curso "Colonialismo, ciencia, tecnología y medicina”, el cual dirigió Diana Obregón, durante el segundo semestre de 1997, en la Facultad de Ciencias Humanas de la Universidad Nacional.
En las últimas sesiones del curso "Colonialismo, ciencia, tecnología y medicina", en el cual se discutieron aspectos fundamentales del proceso de expansión de la ciencia europea y las dinámicas científicas locales en países latinoamericanos, se puso en evidencia un campo de estudio de gran interés que, en los últimos años, ha ganado reconocimiento e importancia en la región. Dicho campo corresponde al estu-

Facultad de Medicina y Facultad de Ciencias Humanas, Universidad Nacional de Colombia, Santa Fe de Bogotá. Recibido para su publicación: 22 de abril de 1998. - Aprobado para su publicación: 30 de abril de 1998. 
dio de las estrategias mediante las cuales las sociedades se enfrentan a las diversas enfermedades y construyen un saber acerca de lo sano y lo enfermo a nivel colectivo o, de manera más general, al estudio de la historia de la salud pública (1).

En los diferentes trabajos que se vienen realizando en la última década, de los cuales sólo analizamos una pequeña muestra en el curso, se distingue el interés por mostrar el campo sanitario inscrito en amplios contextos sociopolíticos, económicos y culturales. Así, en el texto de Nisia Trindade y Nara Britto (2), el interés se dirige al análisis de las concepciones sanitaristas que orientaron a los miembros de la Liga Pro-saneamiento del Brasil a comienzos del siglo $\mathrm{XX}$, para lo cual se elabora un marco político donde se pone de presente el papel fundamental del movimiento nacionalista brasileño. En el documento de Diego Armus (3), política y sanidad se entretejen en una intrincada red de relaciones la cual queda expuesta a partir del análisis del discurso libertario argentino en su vínculo con el saber acerca de la tuberculosis. Diana Obregón, a su vez, muestra el uso político que la profesión médica en Colombia de comienzos del siglo XX hace de los estudios sobre la lepra (4).

Con un enfoque un poco diferente, pero bajo el mismo propósito general antes enunciado, los trabajos de Emilio Quevedo (5) y de María del Pilar Guzmán (6) muestran a la salud pública colombiana dentro de un complejo campo de relaciones donde los intereses económicos y políticos norteamericanos tienen una importancia fundamental y las estrategias de cooperación técnica fundamentan modos de intervención extranjera de gran impacto a la hora de señalar derroteros para la acción.

Siguiendo las directrices formuladas por todos estos trabajos y bajo los efectos de una inicial fascinación por el lúcido esfuerzo desplegado por Marcos Cueto en sus escritos (7-9), el presente artículo intentará mostrar, de manera breve, cómo la salud pública colombiana, al igual que la del resto de Latinoamérica, se desarrolla bajo la profunda influencia de los modelos y las pautas norteamericanas. Sin embargo, también intentará explorar cómo las condiciones particulares hacen del caso colombiano una singularidad inmersa en una tendencia general. Con esto se quiere poner en evidencia el hecho de que, pese a la similitud del influjo que los Estados Unidos tiene en los diferentes países latinoamericanos, la experiencia colombiana es diferente de la peruana, la argentina o la brasileña. Esta diferencia bien puede estar asociada con la ausencia de un significativo movimiento nacionalista de comienzos de siglo que contribuyera a forjar un movimiento sanitarista fuerte y enraizado en lo local y con posibilidades de entablar negociaciones claras y contundentes frente a las posturas norteamericanas, lo que hizo que la salud pública colombiana adoptara una forma diferente y una dinámica diversa a la de otros países de la región.

Esta relativa debilidad de los actores sociales a nivel nacional en el campo sanitario permitirá que, desde muy temprano, la salud pública de corte norteamericano sea el eje para el desarrollo de la salud pública nacional y, posteriormente, bajo la figura del Servicio Cooperativo Interamericano de Salud Pública (SCISP), pueda cumplir la función de orientadora técnica del ministerio y líder en las propuestas de transformación de la educación médica en el país.

Otra disimilitud que puede plantearse entre la experiencia de varios países latinoamericanos y la colombiana es que, en el país, el apoyo extranjero no se articuló fácilmente con los esfuerzos investigativos desarrollados por médicos nacionales en el campo de su especial interés. De hecho, como queda de presente en el trabajo de Diana Obregón, el estudio de la lepra fue primordial para la medicina colombiana; sin embargo, los intereses extranjeros se centraron, en mucho, en el estudio de la anquilostomiasis (uncinariasis), la malaria y la fiebre amarilla ${ }^{1 .}$

\footnotetext{
${ }^{1}$ Esta afirmación puede ser relativizada al atender el caso de la fiebre amarilla en el país. Sin embargo, la conservo como una de las grandes inquietudes que me surgen de la lectura del trabajo de Diana Obregón. Si la investigación en lepra es tan importante para el gremio médico en el país, como ella afirma, entonees los intereses extranjeros relegan este gran interés "nacional". Aparentemente no
} 
En todo caso, lo que evidencian estas diferencias es que aun si los influjos de "políticas colonialistas" se despliegan de manera similar en diversos territorios, las especificidades locales y las tradiciones particulares tienen un papel fundamental que genera procesos diversificados de adecuación, ajuste, transformación o resistencia.

Para dar comienzo a la labor, se intentará establecer una relación del caso colombiano frente al escenario brindado por los países latinoamericanos, ubicando de forma muy general las afinidades que existen entre estos países para luego presentar, de manera sucinta, algunas de las particularidades del caso colombiano. En este sentido, el esfuerzo estará orientado hacia la comprensión de la situación colombiana inscrita en un contexto latinoamericano.

\section{La transformación sanitaria}

A finales del siglo XIX y comienzos del XX, los países latinoamericanos iniciaron un proceso de transformación profunda de sus estructuras sanitarias, motivados por su creciente necesidad de incorporarse al mercado internacional (10). Estas transformaciones condujeron, de manera discontinua en los distintos países, al surgimiento o consolidación de organizaciones estatales de carácter nacional lo suficientemente fuertes como para ser capaces de impulsar acciones rápidas y eficaces en procura de solucionar los grandes problemas sanitarios presentes en la región y, en particular, aquellos que tuvieran algún interés para la economía nacional y el comercio con el extranjero.

La creación de consejos de sanidad, juntas o direcciones nacionales de higiene orientadas a solucionar problemas sanitarios específicos representó, al decir de Juan César García, un cambio notorio con relación al papel jugado anteriormente por el Estado. De una parte, se crearon unidades burocráticas con responsabili-

pasa lo mismo en el Perú donde el estudio de la enfermedad de Carrión tiene una importante atención por parte de investigadores norteamericanos quienes, entre otras cosas, motivaron una fuerte polémica en los círculos médicos peruanos (7); otro tanto pasa en el Brasil con los estudios sobre la uncinariasis. dades ejecutivas en lugar de simples cuerpos colegiados de carácter consultivo y, por otra, se tecnificó el proceso de intervención estatal en salud articulándose con la estructura económica (10).

Argentina, Brasil y Uruguay son los primeros países de América Latina que crean unidades estatales de sanidad a finales del siglo XIX, seguidos por Bolivia, Cuba, Ecuador, Paraguay y Perú, ya a comienzos del siglo XX. En lo referente a Colombia, la Ley 33 de 1913 creó el Consejo Superior de Sanidad dependiente del Ministerio de Gobierno, el cual en 1914 se transformó en la Junta Central de Higiene y, en 1918, se convirtió en la Dirección Nacional de Higiene, dependencia del Ministerio de Instrucción Pública (11). Esta organización sanitaria presente en los países respondió, en gran medida, a la preocupación de las clases dirigentes por superar las cuarentenas marítimas que aumentaban los costos de las importaciones y exportaciones, evitar la introducción de enfermedades del exterior y atender los llamados y exigencias de las potencias comerciales las cuales estaban en ese momento, justamente, negociando acuerdos internacionales para formular convenios y reglamentos sanitarios en miras a reducir al mínimo los requisitos de cuarentena con relación a enfermedades como la peste, la fiebre amarilla, el cólera y la viruela.

Es importante mencionar, siguiendo a Foucault, que en occidente hubo hasta el siglo XVIII e inclusive el XIX, fundamentalmente, dos modelos de organización sanitaria: el primero, suscitado por la lepra, configuró una medicina de exclusión, la cual privilegió como mecanismo "terapéutico" el destierro y la expulsión de los enfermos. El segundo, motivado por la peste, se desplegó como un esfuerzo por distribuir a los individuos uno al lado de otro, aislarlos y vigilarlos. En palabras del mismo Foucault: "Hubo, pues, el esquema médico de reacción a la lepra, que era de exclusión, de exilio, de tipo religioso, de purificación de la ciudad, y el que suscitó la peste, en el que no se aplicaba el internamiento y el agrupamiento fuera del centro urbano sino, por el contrario, se recurría al análisis minucioso de la ciudad, al registro permanente. El modelo 
religioso fue, pues, sustituido por el militar y es la revisión militar y no la purificación religiosa la que en el fondo sirvió esencialmente de modelo a esta organización política médica" (12).

Para el caso de América Latina, las estrategias sanitarias desplegadas por los países a lo largo del siglo XIX estuvieron bastante influidas por estos dos modelos organizativos. El modelo de vigilancia y control, el cual fue instrumentalizado mediante la cuarentena, sirvió como fundamento de la incipiente sanidad portuaria; el otro, matizado por pautas aislacionistas, siguió reglando los dictámenes médicos acerca de la lepra hasta bien entrado el siglo $X X$, por lo menos, en el caso colombiano (4).

A medida que los intercambios comerciales se desarrollaban con una rapidez cada vez mayor, dadas las exigencias de la producción capitalista, la implementación de las cuarentenas, mecanismo efectivo de control sanitario, comenzó a generar grandes dificultades al comercio internacional en la medida en que le colocaba frenos al desarrollo del libre comercio. Esta conciencia de los obstáculos que desde la esfera sanitaria se tenía para el desarrollo comercial, llevó a intensos esfuerzos por establecer una organización sanitaria a nivel mundial y constituir un código sanitario internacional. Es en este proceso donde los países "desarrollados" logran imponer pautas al resto de países, bajo el doble propósito de procurar la salud de los pueblos y regular las posibilidades de intercambio de productos. La realización de las distintas conferencias sanitarias internacionales a partir de 1851, la fundación de la Office International d’Hygiene Publique en 1907 y la creación de la Oficina Sanitaria Panamericana en 1903, serán una muestra de los esfuerzos por construir regulaciones internacionales frente a la salud pública (13).

Para los países latinoamericanos, estos procesos de organización sanitaria internacional implicaron una fuerte exigencia hacia sus estructuras administrativas y retos enormes para sus propias organizaciones sanitarias. Estos retos, si bien movilizaron grandes recursos nacionales, también abrieron la puerta al influjo extranjero, especialmente norteamericano, dadas las precarias condiciones sanitarias, el débil desarrollo técnico en el área y las acciones poco efectivas con las cuales se trabajaba desde tiempo atrás.

Esta apertura permitió que América Latina se convirtiera, por un lado, en un escenario proclive a la cooperación técnica norteamericana y al dominio neocolonial y, por otro lado, en un campo de pruebas de los conceptos y las técnicas desarrolladas por la salud pública estadinense. Como bien lo señala Marcos Cueto: "Entre 1918 y 1940 América Latina se convirtió en un campo de pruebas de uno de los conceptos más ambiciosos y controvertidos de la salud pública moderna: la erradicación de enfermedades" (8).

La Fundación Rockefeller (FR) jugó un papel fundamental no sólo en la aplicación del concepto erradicacionista a través de sus campañas sino también en la reorganización de las instituciones de salud pública latinoamericanas, en la extensión de los servicios sanitarios a las zonas rurales y en la sustitución de los modelos franceses por los norteamericanos como referentes de acción. Para Colombia, la cooperación en salud pública por parte de la Junta Sanitaria Internacional de la FR comenzó en 1917 cuando por solicitud del gobierno nacional una comisión norteamericana se encargó del estudio de la presencia de fiebre amarilla en el territorio nacional. Sin embargo, esta cooperación sólo se concretará de manera definitiva con la campaña en contra de la uncinariasis iniciada en 1919 (5).

\section{Las campañas de erradicación}

Tal como lo señala Marcos Cueto, la FR fijó su atención en tres enfermedades tropicales, las cuales fueron consideradas técnicamente como susceptibles de eliminación mediante campañas directas de corta duración. Estas, por tanto, se convirtieron en focos de erradicación y ejes de acción sanitaria. Dichas enfermedades fueron la anquilostomiasis, la fiebre amarilla y la malaria.

En lo que se refiere a la anquilostomiasis (uncinariasis), la FR entra en América Latina a partir de una evaluación técnica realizada en 
Brasil en 1916. Posteriormente, se desarrollan campañas en Colombia (1920-1935), Paraguay (1923-1927), Venezuela (1927-1928) y México (1923-1928). Los gobiernos latinoamericanos acogieron y apoyaron estas campañas las cuales fueron percibidas, en palabras de Cueto, "como una oportunidad para terminar con el dualismo en la administración de los servicios de salud entre el Estado y las municipalidades, característico de la organización de la salud pública de varios países latinoamericanos en la década de 1910" (8).

A pesar de extenderse durante unos años más el trabajo en las campañas, a mediados de los años, veinte la FR decidió abandonar los programas de erradicación de la anquilostomiasis y convertirlos en servicios generales de salud rural. Este hecho lo refiere Fred Soper en uno de sus escritos acerca de la lucha contra la uncinariasis en Paraguay:

"En la primera parte de 1923, unos seis u ocho meses antes de la fecha en que yo iba a comenzar el programa de anquilostomiasis en $\mathrm{Pa}$ raguay, la fundación decidió poner término a los programas de lucha contra esa enfermedad y convertirlos en servicios generales de salud rural, medida que me causó gran sorpresa. Aunque esta política no era de aplicación inmediata, la suerte estaba echada y era evidente que en la fundación no había posibilidades para dedicarse toda la vida a la prevención de la anquilostomiasis" (14).

Entre los motivos señalados por Cueto que justifican este cambio, cabe mencionar los siguientes: la gigantesca dimensión de esta enfermedad en algunos países, el elevado costo de las campañas, la resistencia de curanderos y algunos médicos, las tensiones entre los expertos extranjeros y los inspectores locales, la efímera existencia de las letrinas, las complicadas condiciones políticas de ciertos países y la dificultad para convencer a los funcionarios locales de que la anquilostomiasis era un problema nacional (8).

En Colombia, en 1920 se crea el Departamento de Uncinariasis el cual inicia actividades en la zona cafetera donde la enfermedad era endémi- ca. Las actividades con las cuales inicia labores este departamento se orientaron, básicamente, hacia el diagnóstico y tratamiento masivo de la población y hacia el saneamiento, mediante la construcción de letrinas en las viviendas de los campesinos (5).

Frente a la fiebre amarilla, en 1916, la FR inició estudios para explorar la situación de esta enfermedad en Suramérica (14) y, en 1918, realizó su primera campaña en América Latina al controlar un brote epidémico en el puerto de Guayaquil (9). Las actividades realizadas produjeron tanto optimismo que se reconoció desde muy temprano que, en el decenio de los años veinte, la fiebre amarilla había desaparecido de las estadísticas en todos los países infectados en los que la fundación había emprendido campañas de erradicación (14). El ambiente de optimismo así generado descansó, tal como lo señala Cueto, en dos creencias científicas que a la postre resultaron erradas: la teoría de los centros clave y el desarrollo de la vacuna y el suero de Hideyo Noguchi.

La teoría de los centros claves consideraba que la fiebre amarilla era una enfermedad humana que se desarrollaba en los núcleos urbanos, producía inmunidad en las personas que sobrevivían a la primoinfección y era transmitida exclusivamente por el Aedes aegypti. De igual manera, la teoría asumía que la enfermedad era endémica en puertos y ciudades costeras. Esta comprensión epidemiológica postulada por la teoría de los centros claves y los hallazgos de Noguchi, quien creyó encontrar el germen de la fiebre amarilla al cual le llamó Leptospira icteroides, produjeron la sensación de que el conocimiento necesario para la erradicación de la enfermedad estaba a la mano. Esto fundamentó el optimismo del momento.

Otras campañas y actividades contra la fiebre amarilla en la región se llevaron a cabo en Guatemala (1919), Perú (1920-1922), Colombia (1923), Brasil (1923-1940) y México (19211923). Pese al entusiasmo desplegado en cada una de estas actividades y al deseo manifiesto por lograr la erradicación, nuevos brotes epidémicos aparecieron en la región a partir de 1928. En los años treinta, la FR comenzó a perder in- 
terés en la fiebre amarilla como un problema epidemiológico y de administración sanitaria. Pérdida de interés que puede relacionarse, entre otras cosas, con el surgimiento de una inclinación nueva por los estudios de laboratorio. Al decir de Soper: "El programa de la fundación para combatir la fiebre amarilla en las Américas en el período de 1918 a 1928 había sido esencialmente administrativo, dedicado en gran parte a reducir los criaderos de mosquitos en centros antes endémicos y en pequeñas comunidades hasta un nivel inferior al que permitía a la enfermedad perpetuarse. Los destacados resultados de la investigación de la fiebre amarilla en Africa intensificaron el interés de la Fundación en las investigaciones de laboratorio; en unos pocos años dejó de dedicarse únicamente a cortar la distancia entre los conocimientos existentes y su aplicación en beneficio de la población mundial" (14).

En Colombia, entre 1916 y 1917, la comisión de la fiebre amarilla de la FR, formada por William C. Gorgas, Henry Carter, Juan Guiteras y Theodore Lyster entra al país por Buenaventura en viaje hacia Bogotá. Dentro del país, los miembros de la comisión se enteran de casos informados en Buenaventura, Barbacoas, Caldas, Honda y, especialmente, Muzo. Después del examen del litoral Caribe y de algunas averiguaciones adicionales, la comisión concluye que en Colombia no existen centros endémicos de fiebre amarilla (14). Sin embargo, nuevos brotes epidémicos en los años treinta llevaron al estudio de casos de fiebre amarilla rural estableciéndose la existencia de lo que se ha conocido como fiebre amarilla selvática.

En lo que hace relación con la malaria, la participación de la FR en los países latinoamericanos inició con las primeras campañas en Brasil (1918-1928), Argentina (1925) y Venezuela (1927-1928). En estos años, la labor fundamental de la FR se concentró en convencer a las autoridades locales de la real posibilidad de control de la enfermedad y en el entrenamiento de personal para los programas antimaláricos, actividades que en Colombia se desarrollaron a partir de los años treinta.

\section{La salud pública colombiana made in USA}

Como se mencionó anteriormente, el comienzo del siglo XX trajo para Colombia la exigencia de transformar sus estructuras económicas y sanitarias en miras al reto que le planteaba su vínculo con los mercados internacionales. Así, la necesidad de establecer una organización sanitaria estatal se presentó ligada a la economía cafetera, la cual creó una burguesía en alianza con el capital monopólico y requirió de un manejo adecuado de los puertos marítimos y fluviales con el fin de restarle obstáculos a los intereses agroexportadores (10).

Se puede decir que el principal objetivo de los cambios establecidos en la estructura sanitaria en el país desde 1913 y, en particular, la creación de la Dirección Nacional de Higiene fue el de superar las trabas de una organización sanitaria arcaica que, entre otras cosas, estaba a cargo de la policía y era sumamente inefectiva en el manejo de las epidemias y en el control de las enfermedades de la población trabajadora. Dicha dirección se ubicó bajo el control del Ministerio de Instrucción Pública, lo cual significó que se asumía a la higiene pública como una de las funciones educativas del Estado. Sin embargo, en 1920, la dirección se traslada al Ministerio de Agricultura y Comercio, evidenciándose así el vínculo existente entre la sanidad y la economía (11).

En este proceso de constitución de una estructura sanitaria de carácter nacional, los intereses norteamericanos entraron en juego de manera muy rápida. De hecho, la colaboración técnica prestada por la Junta Sanitaria Internacional de la FR empezó en 1917. En ese año, como ya se ha mencionado, el Instituto Rockefeller, por solicitud del gobierno colombiano, hizo un estudio para determinar la presencia de fiebre amarilla en el país, cuyo propósito último fue definir si era necesario mantener la cuarentena impuesta por la Oficina Sanitaria Panamericana a todos los barcos colombianos y a todos aquellos que tocaran nuestras costas (5).

En 1919, una nueva solicitud del gobierno nacional a la Junta Sanitaria Internacional para iniciar 
la campaña de erradicación de la uncinariasis, vincula de manera directa a la FR en el desarrollo de la salud pública colombiana. Es interesante señalar que los cambios en la política norteamericana hacia los países productores de café se reflejan en los programas de erradicación de la uncinariasis. Las mayores inversiones en estos programas se realizaron en las zonas cafeteras del Brasil en 1920 las cuales fueron declinando hacia 1923-1926 coincidiendo con la promulgación de la Ley de Protección del Productor del Café y el préstamo concedido por Inglaterra al gobierno brasileño. A su vez, en 1926, el año en que el presidente Hoover propuso que se establecieran nuevas fuentes de abastecimiento de café, se acrecentó la inversión en los programas de erradicación de la uncinariasis en Colombia (15).

La cooperación de la FR también se efectuó a partir de trabajos dirigidos a la erradicación de la fiebre amarilla en Bucaramanga (1922-1923), al estudio de la malaria (1932), a la creación del Laboratorio de Higiene Pública de Barranquilla y la escuela de inspectores (1933), al estudio de la fiebre amarilla en zonas rurales y selváticas (1933) y a la implementación de la Unidad Sanitaria de Pereira (1934) (6).

Frente a las asignaciones presupuestales hechas en Colombia por la División de Salud Internacional de la FR entre 1913 y 1940, merece señalarse que se destinaron US\$179.929 para las acciones en contra de la anquilostomiasis, US\$245.276 para las acciones en contra de la fiebre amarilla, US $\$ 19.353$ para las acciones en contra de la malaria y US $\$ 68.280$ para servicios de salud pública y educativos (8).

Con las ayudas prestadas por la FR, los referentes norteamericanos en salud pública tuvieron un influjo determinante en la creación de los modelos sanitarios institucionales adoptados por los gobiernos nacionales. Este influjo estará presente en el establecimiento de las prioridades nacionales, en la orientación de las políticas y los planes sanitarios y en la formación del recurso humano en salud pública.

Frente a los dos primeros puntos, las campañas y las acciones emprendidas con apoyo de la FR buscaron, por un lado, orientar las estrategias de acción sanitaria del país vinculando los intereses extranjeros con los propósitos de desarrollo nacional y, por otro lado, favorecer la reorganización de la estructura sanitaria. Esta reorganización se manifestó en la instauración de servicios independientes por enfermedad, en la ampliación de los servicios sanitarios a áreas rurales y la centralización estatal de la autoridad sanitaria. En lo referente al tercer punto, es importante señalar el papel trascendental que jugó el otorgamiento de becas de estudio en salud pública en universidades estadinenses, estrategia que sirvió para formar a los futuros funcionarios de la salud pública en el país, quienes cumplieron la función de consolidar el modelo sanitarista norteamericano en Colombia.

Es importante señalar que en el período correspondiente a los años anteriores a la Segunda Guerra Mundial, del cual nos hemos ocupado en este escrito, el influjo norteamericano se dio a través de las fundaciones privadas norteamericanas, en particular la FR. Sin embargo, en años posteriores, en el contexto de la guerra y bajo las nuevas políticas norteamericanas con relación a los países latinoamericanos, en especial con la política del buen vecino del presidente Roosevelt, se dio un viraje fundamental a la acción de cooperación técnica entre Estados Unidos y Colombia la cual, mediante el Instituto de Asuntos Interamericanos, entidad gubernamental con sede en Washington, generó nuevos programas y proyectos.

En este sentido, el influjo norteamericano, que en un primer momento se efectuó mediante acciones del sector privado, se canalizó a través de instancias gubernamentales estadounidenses, conformando un vínculo más expedito de intereses entre dos naciones.

En el marco de esta política del buen vecino, se lleva a cabo en Río de Janeiro, en 1942, la tercera reunión de consulta de Ministros de Relaciones Exteriores de las repúblicas americanas. Allí se plantea como objetivo general, la protección del hemisferio occidental y el estudio de medidas para restablecer el orden mundial destrozado por la guerra. Para dar cumplimiento a este objetivo, se establecen como puntos de 
la agenda de discusión la solidaridad económica, la ruptura de relaciones con Alemania y sus aliados, la producción de materiales estratégicos para la confrontación bélica, el desarrollo del intercambio comercial y el mejoramiento de la salud y la salubridad pública (6).

Después de esta reunión, se estableció un programa cooperativo entre el instituto de Asuntos Interamericanos (IAI) y las repúblicas americanas para implementar un plan de salubridad y saneamiento, el cual, más que responder a un diagnóstico concienzudo de los problemas específicos de la región, buscó contrarrestar cualquier posible influencia que pudieran tener los países del eje Berlín-Roma-Tokio en los países latinoamericanos.

El gobierno colombiano inició, de manera muy rápida, los trámites pertinentes para poner en ejecución el programa de cooperación antes referido y, después de recibir la visita de Nelson Rockefeller, en ese momento coordinador de la Oficina de Relaciones Interamericanas del IAI, autoriza la creación del Servicio Cooperativo Interamericano de Salud pública (SCISP) en 1942 y, en 1943, se crea como organismo dependiente del Ministerio de Trabajo, Higiene y Previsión Social (11).

Con el progresivo desarrollo de SCISP, el cual se convirtió en una especie de ministerio de salud pública dentro del Ministerio de Salud Pública, se consolidó un proceso de influencia de la salud pública norteamericana en la estructura sanitaria colombiana que comenzó desde los albores del siglo XX. Proceso que, en sus comienzos, no contó con un movimiento nacionalista fuerte que posibilitara la construcción de modelos alternos frente a los referentes norteamericanos vehiculizados por la FR como, según el relato de Anne-Emanuelle Birn, sí aconteció en México (16).

Esta ausencia de un fuerte movimiento nacionalista también impidió, a su vez, el desarrollo de una escuela tropicalista al estilo de la estudiada por Julyan Peard en el Brasil del siglo XIX y el desarrollo de una tradición bacteriológica como la referida por Marcos Cueto en el Perú de comienzos de siglo (7). Sin pretender afirmar que aquí en Colombia se implantaron los esquemas salubristas propuestos por la FR -y por la Escuela de Higiene y Salud Pública de Johns Hopkins que le sirve de sostén - en su totalidad y sin ninguna dificultad, interesa dejar como hipótesis de trabajo, después de esta sucinta revisión, que los procesos de negociación con dichos modelos son precarios, dada la ausencia de una fuerte tradición sanitaria nacional, y que los procesos de resistencia se dan más por la vía de las disposiciones cotidianas de la vida de las poblaciones -y de algunos médicos particulares- que pur una dinámica de construcción de un modelo alterno propiciado por una comunidad académica comprometida en pensar en torno a los grandes problemas sanitarios del país.

\section{Referencias}

1. Cueto M. Introducción. En: Cueto M, editor. Salud, cultura y sociedad en América Latina. Nuevas perspectivas históricas. Lima: IEP/OPS; 1996. p. 13-30.

2. Trindade N, Britto N. Salud y nación: propuesta para el saneamiento rural. Un estudio de la revista Saudé 1918-1919. En: Cueto M, editor. Salud, cultura y sociedad en América Latina. Nuevas perspectivas históricas. Lima: IEP/OPS; 1996. p. 135-58.

3. Armus D. Salud y anarquía: la tuberculosis en el discurso libertario argentino, 1870-1940. En: Cueto M, editor. Salud, cultura y sociedad en América Latina. Nuevas perspectivas históricas. Lima: IEP/OPS; 1996. p. 111-33.

4. Obregón D. De "árbol maldito" a "enfermedad curable": los médicos y la construcción de la lepra en Colombia, 1884-1939. En: Cueto M, editor. Salud, cultura y sociedad en América Latina. Nuevas perspectivas históricas. Lima: IEP/OPS; 1996. p. 159-78.

5. Quevedo E. Políticas de salud o políticas insalubres? De la higiene a la salud pública en Colombia en la primera mitad del siglo XX. Biomédica 1996;16:345-60.

6. Guzmán, MP. Cooperación técnica norteamericana en salud pública 1900-1953 (tesis). Santa Fe de Bogotá: Universidad Externado de Colombia; 1996.

7. Cueto M. Tradición médica, bacteriología e intervención extranjera en el Perú. En: Excelencia científica en la periferia. Lima: Concytec; 1989. p. 119-52.

8. Cueto M. Los ciclos de la erradicación: la Fundación Rockefeller y la salud pública latinoamericana, 19181940. En: Cueto M, editor. Salud, cultura y sociedad en América Latina. Nuevas perspectivas históricas. Lima: IEP/OPS; 1996. p. 179-201. 
9. Cueto M. Sanidad desde arriba:la fiebre amarilla, la costa norte y la Fundación Rockefeller. En: El regreso de las epidemias. Salud y sociedad en el Perú del siglo XX. Lima: IEP; 1997. p. 59-86.

10. García JC. La medicina estatal en América Latina. En: Pensamiento social en salud en América Latina. México: Interamericana McGraw-Hill, OPS; 1994. p. 95-143.

11. Quevedo E, Hernández M, Miranda N, Mariño C, Cárdenas $\mathbf{H}$, Wiesner $\mathbf{C}$. La salud en Colombia: análisis sociohistórico. Bogotá: Ministerio de Salud, DNP; 1990.

12. Foucault M. Historia de la medicalización. Educación médica y salud 1977;11(1):3-25.

13. Howard-Jones, N. Problemas de organización de la salud pública internacional entre las dos guerras mundiales. Crónica de la OMS 1977;31:435-48.
14. Soper FL. Andanzas por el mundo de la salud. OPS/ OMS. Publicación científica No.355. Washington. 1979.

15. García, JC. La enfermedad de la pereza. En: Pensamiento social en salud en América Latina. México: Interamericana McGraw-Hill, OPS; 1994. p. 150-71.

16. Birn, AE. Las unidades sanitarias: la Fundación Rockefeller versus el modelo Cárdenas en México. En: Cueto $M$, editor. Salud, cultura y sociedad en América Latina. Nuevas perspectivas históricas. Lima:IEP/OPS; 1996. p. 203-33.

17. Bowers J, Purcell E. Informe de William Henry Welch y Wickliffe Rose a la Fundación Rockefeller (apéndice). En: Escuelas de salud pública: presente y futuro. Buenos Aires: Editorial El Ateneo; 1976. p. 166-74. 